\author{
Proceedings of 15th IEEE/ASME International Conference on Mechatronic and Embedded \\ Systems and Applications \\ MESA 2019 \\ August 18-21, 2019, Anaheim, USA
}

\title{
IDETC2019-97526
}

\section{A CLOUD-BASED HEALTHCARE INFRASTRUCTURE FOR NEONATAL INTENSIVE-CARE UNITS}

\author{
Lucia Migliorelli;, Annalisa Cenci, Michele Bernardini, \\ Luca Romeo, Sara Moccia, Primo Zingaretti \\ VRAI Laboratory \\ Department of Information Engineering \\ Università Politecnica delle Marche \\ Ancona, Italy \\ Email: I.migliorelli@pm.univpm.it, a.cenci@pm.univpm.it, \\ m.bernardini@pm.univpm.it, I.romeo@univpm.it, \\ s.moccia@univpm.it, p.zingaretti@staff.univpm.it
}

\begin{abstract}
Intensive medical attention of preterm babies is crucial to avoid short-term and long-term complications. Within neonatal intensive care units (NICUs), cribs are equipped with electronic devices aimed at: monitoring, administering drugs and supporting clinician in making diagnosis and offer treatments. To manage this huge data flux, a cloud-based healthcare infrastructure that allows data collection from different devices (i.e., patient monitors, bilirubinometers, and transcutaneous bilirubinometers), storage, processing and transferring will be presented. Communication protocols were designed to enable the communication and data transfer between the three different devices and a unique database and an easy to use graphical user interface (GUI) was implemented. The infrastructure is currently used in the "Women's and Children's Hospital G.Salesi" in Ancona (Italy), supporting clinicians and health opertators in their daily activities.
\end{abstract}

\section{INTRODUCTION}

Preterm birth affects the anatomical and functional development of all infant's organs and is one of the primary causes of neonatal mortality. Intensive medical attention of patients with

\footnotetext{
${ }^{*}$ Address all correspondence to this author.
}

critical organs immaturity is crucial to avoid the development of short- and long-term complications [1].

Within neonatal intensive care units (NICUs), cribs are equipped with electronic devices aimed at: monitoring, administering drugs and supporting clinicians in making diagnosis and administering treatment. Each device has its own interface and clinicians visually inspect data from each device separately to take decisions. Then data are manually stored within a computer in the NICU and eventually sent to a local server (i.e., within the hospital) [2,3]. This practice is slow, laborious and susceptible to mistakes. Moreover, an important delay between data generation, availability and accessibility to the medical staff arises $[2,4,5]$.

In this complex scenario, a system to automatize data storage, processing and transmission may improve the diagnostic and therapeutic process and, more in general, the quality of care delivery. A straightforward solution could be the development of a dedicated Health Information System (HIS) to store data acquired in the NICU in the form of Electronic Medical Records (EMRs). A HIS that manages these EMRs should meet three main demands: (i) integration of data from different sources (e.g. hospitals, private clinics, health care professionals), (ii) correct data acquisition and (iii) support for clinical decisions. In order to achieve this goal, however, the EMR should also have in-
Copyright (c) 2019 by ASME 
teroperability characteristics (i.e., EMRs from different devices should communicate with each other), since it processed data come from different sources and produced autonomously [6,7].

This paper proposes a cloud oriented architecture to automatize the process of crib-EMR gathering and to allow medical staff to access data remotely. Medical communication protocols and proprietary software are designed to automatically enable the communication between devices and the EMR transfer from the crib node to a EMR database (i.e., a database collecting EMR from different infants). To facilitate data visualization a graphical user- friendly interface is proposed, too. Furthermore, decisionmaking tools are designed and implemented to support clinicians in performing monitoring actions and diagnosis and administering treatment.

This paper is organized as follows: Sec. "BACKGROUND AND MOTIVATION" introduces the clinical background and the main motivation of this work. Sec. "CLOUD-BASED INFRASTRUCTURE" presents the overall cloud-based architecture. Preliminary results are presented in Sec. "RESULTS" and discussed in Sec. "DISCUSSION", which also concludes this paper giving future perspectives of this work.

\section{BACKGROUND AND MOTIVATION}

With the aims at fostering and improving the storage and sharing of health data, Italian universities, research centers and hospitals are cooperating to develop HIS for collecting EMRs in cloud with the goal to integrate Electronic Health Records (EHRs). In fact, EHRs and EMRs are different concepts, often mentioned interchangeably, as an EMR is a narrower view of the patients' anamnesis, normally collected within a single structure, while EHR is a more comprehensive report of patient's clinical history [8].

EHR can be then used for a triple purpose:

1. Prevention, diagnosis, treatment and rehabilitation;

2. Study and scientific research in the medical context;

3. Health planning, verification of the quality of care and evaluation of healthcare.

Marche Region is particularly active in disseminating the guidelines to construct regional EHR infrastructures to facilitate future suppliers of systems that will interact with the regional EHR in the implementation of dedicated integration interfaces [9]. New regional standards will bring to the creation of a network which will include all neonatal care units of the Marche Region. This would facilitate data sharing between structures and make easier patient transferring within hospitals of the network, ensuring care continuity during the hospitalization.

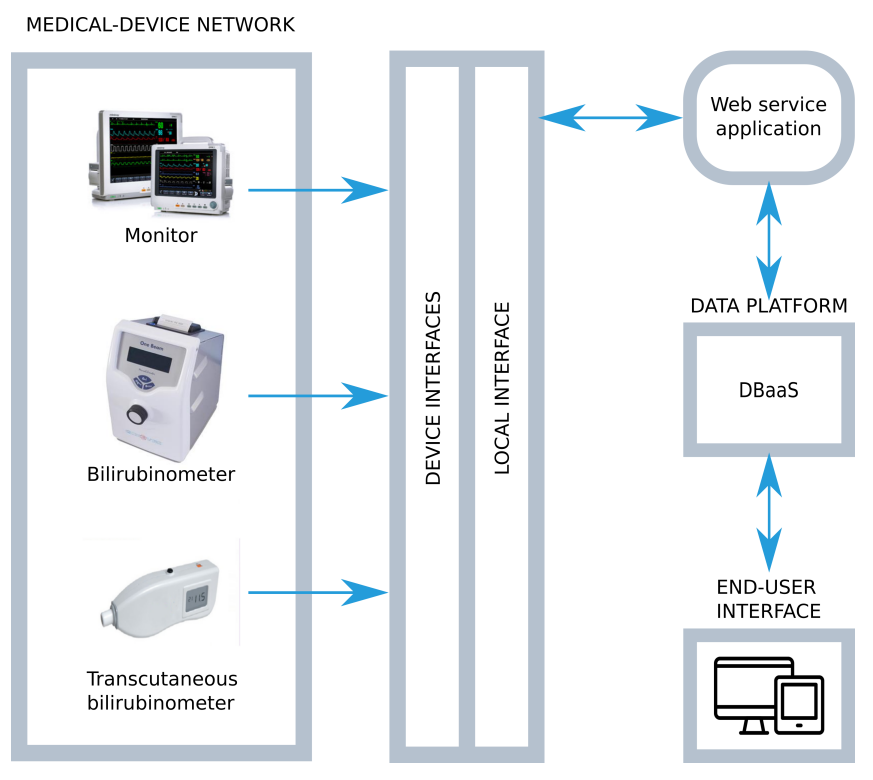

FIGURE 1. The cloud-based healthcare infrastructure

\section{CLOUD-BASED INFRASTRUCTURE}

Figure 1 shows the cloud-based architecture for crib EMR collection, storage, processing, distribution and visualization. A preliminary version of the architecture was described in [3], where bilirubinometer was the only device considered.

The main components of the architecture are: (i) data platform, (ii) medical device network, (iii) end-user interface

\section{Data platform}

Without the need of setting up a physical hardware and installing and maintaining a software, database-as-a-service (DBaaS) allows to access easily the capabilities of a productionready relational database, optimized in terms of performance and with zero data redundancy. Scalability is an intrinsic property of the database whose storage resources are automatically updated as the database increases in volume. Moreover, DBaaS includes automated backups, database snapshots and automatic host replacement [10]. For what concern data security, DBaaS ensures data encryption both during the transit (using Secure Sockets Layer (SSL)) and during the cloud storage phase [11]. Besides, many tools of the solution for secure data sharing and service interoperability presented in [12] are implemented in the actual system. Particularly, in accordance with recent laws on personal-data protection and treatment, the following measures were adopted:

1. Computer authentication;

2. Adoption of procedures for the management of authentication credentials;

3. Use of a system of authorization; 
4. Periodic update of the identification of treatment allowed to individual officers and employees for management or maintenance of electronic instruments;

5. Protection against illegal treatment of data, unauthorized access and certain computer programs;

6. Adoption of procedures for storing backups, restoring access data and systems;

7. Adoption of encryption techniques or identification codes for certain data processing operations, carried out by health organizations, which reveal health status or sexual life.

Under these conditions, all collected EMRs are protected with a "strong" encryption system that guarantees a high degree of security; the assumption is that only the rightful owner - the licensed doctor - has access to the data of his competence through a single sign on (SSO) authentication system.

\section{Medical-device network}

To construct a proper network of medical devices, drivers for communicating with the input devices and acquiring data were developed in Python. They work in a Linux environment, but they were tested also on Windows OS.

Three monitoring instruments located in the NICU of the "Women's and Children's Hospital G.Salesi" were considered:

1. Patient monitor

2. Bilirubinometer

3. Transcutaneous bilirubinometer

\section{Patient monitor}

Patient monitors, commonly called cardiac monitors, control heart rhythm, breathing rate, partial oxygen, carbon dioxide pressures in the bloodstream and temperature. Each parameter is processed by a specific sensor that, via a set of amplifiers and circuits, allows the visualization of the parameter trend on the screen.

Patient's monitor used in the NICU of "G.Salesi" hospital is the Datascope Passport 2, which detects the following parameters:

1. Oxygen saturation, $\mathrm{SaO} 2[\%]$

2. Heart rate detected by the saturimeter [bpm]

3. Heart rate detected by the electrocargiographic signal(ECG) [bpm]

4. Respiratory rate [apm]

5. Systolic, diastolic and average pressures $[\mathrm{mmHg}]$

6. Esophageal temperature [C]

Each monitor has an alarm system to alert medical staff when a parameter is out of the physiological ranges.

\section{Bilirubinometer}

Monitoring bilirubin levels is essential as the aggravation of neonatal jaundice (caused by hyperbilirubinemia) may bring to kernicterus, which irreversibly affects patients' neurophysiological functions.

The bilirubinometer used in the NICU of "G.Salesi" hospital is the Ginevri One Beam, which is an invasive biomedical instrument designed to measure accurate Total Serum Bilirubin (TSB) concentration. The device filtering system allows to project a single optical beam onto a fixed point of the blood sample. Once the light beam crosses the sample, it is cleaned of any disturbing frequencies to have a more stable signal. Then advanced signal processing techniques allow to achieve the displayed infant's bilirubin level.

\section{Transcutaneous bilirubinometer}

TSB measure is the current gold standard for evaluating neonatal jaundice. However, invasive blood sampling has its drawbacks: it is painful and stressful for the infant, blood loss may bring to an increased risk for osteomyelitis and the site of sampling may be prone to infections [13,14].

Transcutaneous bilirubinometry, on the other hand, is a noninvasive and painless method that provides an instantaneous read-out of the cutaneous bilirubin concentration (TcB). Transcutaneous bilirubinometry is based on optical spectroscopy and it relates the amount of light absorption by bilirubin (i.e., the yellow color of the skin) to the concentration of bilirubin in the skin.

The transcutaneous bilirubinometer used in the NICU of "G.Salesi" hospital is the Gräger JM-105. It projects white light into the skin (of the forehead or sternum of the baby) and measures the intensity of the returning wavelengths. The optical spectrum of signal reflected by newborn's subcutaneous tissues, shows hemoglobin, dermal maturity, melanin and bilirubin. Signal processing techniques, knowing the spectral characteristics of hemoglobin, dermal maturity and melanin, allow to assess the bilirubin level. The Gräger JM-105 has an integrated barcode scanner that can automate data entry for storing patient and nurse ID, medical record number, patient name and date of birth.

\section{End-user interface}

The end-user web interface was built using the following choices:

1. PHP as the server side scripting language;

2. JavaScript as the client side scripting language;

3. AngularJS for the developing of the client side Web application.

The interface is connected to the cloud database through Web Services (WS) in a Service-Oriented Architecture (SOA). It consists of a cross-platform solution that can be used with Windows, Linux iOS and Android computers by maintaining the same user interface. It is a simple and user-friendly interface 
based on the touch screen paradigm to minimize the necessary interactions to access information and functions. In addition, unlike the traditional EMRs, mostly based on "pull" logic and where the user needs to know what to look for, it adopts "push" logic by which the system suggests to the user what to see and what actions to carry out on the basis of rules and clinical protocols.

\section{Authentications, authorizations and data access}

As previously introduced, the authentication is ensured by SSO. In particular, an algorithm is defined to establish the authorization (user and password) to access data. The generation of credentials can be activated from website, where one can also access the procedures for password renewal. Credentials provided during the registration are activated after the physician has agreed to a procedure sent via email. Physicians with the access key may consult and modify data and their movements are fully tracked to ensure data integrity.

Also infants' parents can request authentication credentials to access an area made available directly by clinicians. In this area, parents can reconstruct the "Patient Summary" of their children and update clinically relevant data following doctor's guidelines.

\section{Applications, tools and services}

The web interface contains several tools and applications. Each tool was developed with the support and supervision of the NICU medical staff of the "G.Salesi" hospital in Ancona. These applications interact with the database through the Web Services, supporting the clinician in his/her professional activity, encouraging the improvement of his/her clinical practice and facilitating care delivery.

To improve data collection quality benchmarking activities and self-audit access systems are available too. Although the platform makes frequent backups automatically, disaster recovery was implemented allowing full restoration of the database in real time, lowering users responsibility burden.

Different tools were implemented in the platform, the three main ones are:

The “Anagraphic Tool”, which allows to collect patient' s anagraphic information, relevant for his/her management. Immediately after birth, the newborn infant is registered and identified with a unique code that will be used during the hospitalization. All the sensitive data are encrypted and gathered in an independent personal database. With the aim of reconstructing patients' history, this tool has a module for the temporal tracking of all data.

The "Growth Tool", which allows to insert all data on patient's growth during hospitalization. The data collected are: weight (once a day), length and head circumference (once a week). Regularly, notifications are sent to the medical staff to remind them measurements collection. During data entry phase, algorithms to compare the inserted data with the previous ones were developed. Warning alarms were included to inform medical staff of any changes. For example, in the case of weight, if the new measurement exceeds the previous one by more than $5 \%$, the person who is filling the database receives an alarm; if it exceeds $7 \%$, soon a notification to repeat the measurement appears. Other growth-related information such as tibia length, forearm length and skin folds can be added and displayed on another web page.

The "Nutrition Tool", which ensures that the infant has all the essential substances to complete his/her development. Generally, the nutrition of preterm infants can be: (i) parenteral, (ii) gavage (iii) enteral.

The most premature babies can not accept or digest milk so they are fed intravenously (i.e., bypassing the digestive tract) with parenteral nutrition. Parenteral nutrition implies the intravenous administration of a solution enriched of proteins, vitamins, minerals, sugar, fat, water and drugs. Once the baby is stable enough to receive feeding, he/she is fed via gavage nutrition. With gavage nutrition breast milk is administered with a small tube which runs from the nose directly to the stomach. Enteral nutrition is indicated for infants with a developed sucking- deglutition mechanism. Enteral nutrition consists of feeding the baby directly via mouth with a solution of fortified breast milk with protein, fat, calcium, phosphorus and sodium or, alternatively, milk in a specific formula directly by mouth. The "Nutrition Tool" consists of an algorithm that, for each patient, evaluates the daily amount of nutrients necessary for a proper development. This algorithm was implemented starting from data collected in the "Anagraphic" and "Growth" tools (i.e.,life days, current GA, weight...) and on the basis of nutritional schemes created by the head physician of "G. Salesi" Hospital together with a nutrition specialist.

\section{RESULTS}

To evaluate system performance and usability, quantitative and qualitative analysis were conducted. For quantitative evaluation the simulated - load testing interface SOAPUI ${ }^{1}$ was used. As previously reported in [3], the database average traffic per hour [MByte/hour] was 471 (received) and 832 (sent). The database average connections per hour were 578 (max contemporary connections) and 0 (failed connection attempts). Average query per hour was $386 \mathrm{k}$ (select), 36 (insert), 27 (update), 8 (delete).
${ }^{1}$ https://www.soapui.org 
As recommended in [15], preliminary usability tests were conducted both for proving the advantages of integrating the three electronic devices (i.e., patient monitor, bilirubinometer and transcutaneous bilirubinometer) and for testing the web interfaces. These tests were proposed to 6 physicians and 4 nurses, after signing a written consent for privacy issues.

The integration of the three medical devices within a unique infrastructure attracted broad support from the clinical staff. Hence it ensured immediate availability of patients' personal data and improved considerably data sharing among medical staff.

Regarding the web- interface usability tests, experts responsible of the study provided each participant with a website name. Then each participant was asked to explore autonomously the web interface, to compile a short survey on system usability and to annotate comments. Observation metrics were calculated and the results showed an overall satisfaction (more than $85 \%$ of surveyed people) in system usability. Some comments arose on button positioning and icons meaning. Another test was performed asking participants to complete different tasks autonomously (e.g., making an electronic prescription (e-prescription)). Average time to complete a single task was of about 26 seconds. To prove the effectiveness of the touch interface, the same set of tests was performed using a tablet. A survey revealed that the $76 \%$ of users preferred using the tablet.

\section{DISCUSSION}

This paper presented a cloud-based healthcare infrastructure, already in use in the "G. Salesi" hospital of Ancona, for extracting, collecting and analyzing preterm infants' EMRs, acquired via different devices.

An intuitive and easy-to-use web interface was created for visualizing temporally ordered patients' data using graphs and tables, and specific tools (i.e., the "Anagraphic Tool",the "Growth Tool" and the "Nutrition Tool") were implemented to support the medical staff in care delivering.

All data are encrypted and access to data is controlled (i.e., allowed only to those who have the necessary authorizations) and differentiated in relation to the role played within the hospital. Also infants' parents have the key access to visualize infants' clinical history and report of the various tools and to make annotations on baby's health status. With a strong focus on cybersecurity all the authorized members have remote access to the cloud database and may analyze patients' data everywhere both via pc and mobile devices (i.e., tablets and smartphones). This data organization allows: clinicians to visualize real- time and contemporary vital parameters from different electronic instruments (i.e., patient monitor, bilirubinometer, transcutaneous bilirubinometer) and to make correlations within them; "transversal" interoperability among different specialists, ensuring patients' continuity of care and it permits to baby's parents to understand their child's clinical condition, making them active part of the baby's treatment. Future developments may involve the integration of the information coming from other devices already implemented, i.e., a medical imaging system to estimate the preterm infant respiratory rate [16] and a video-based system for preterm infant's movement assessment by some important indicators [17], as well new tools for measuring vital parameters without contact and a tele-consulting platform to assist parents in children's care after hospital discharge.

In conclusion, the results obtained prompt the possibility of spreading this system at a national level with a relevant impact on the health system and on clinicians' everyday activities, in terms of: care efficiency and effectiveness and a reduction of social costs of public care.

\section{Acknowledgement}

This work was supported by the European Union through the POR-FESR grant SINC "System Improvement for Neonatal Care"

\section{REFERENCES}

[1] Giuliani, F., Cheikh Ismail, L., Bertino, E., Bhutta, Z. A., Ohuma, E. O., Rovelli, I., Conde-Agudelo, A., Villar, J., and Kennedy, S. H., 2016. "Monitoring postnatal growth of preterm infants: present and future-3". The American Journal of Clinical Nutrition, 103(2), pp. 635S-647S.

[2] Chang, V., and Wills, G., 2016. "A model to compare cloud and non-cloud storage of big data". Future Generation Computer Systems, 57, pp. 56-76.

[3] Cenci, A., Liciotti, D., Ercoli, I., Zingaretti, P., and Carnielli, V. P., 2016. "A cloud-based healthcare infrastructure for medical device integration: The bilirubinometer case study". In MESA 2016- 12th IEEE/ASME International Conference on Mechatronic and Embedded Systems and Applications, no. 7587125, IEEE, pp. 1-6.

[4] Millett, E. R., Quint, J. K., De Stavola, B. L., Smeeth, L., and Thomas, S. L., 2016. "Improved incidence estimates from linked vs. stand-alone electronic health records". Journal of Clinical Epidemiology, 75, pp. 66-69.

[5] Roukema, J., Los, R. K., Bleeker, S. E., van Ginneken, A. M., van der Lei, J., and Moll, H. A., 2006. "Paper versus computer: feasibility of an electronic medical record in general pediatrics". Pediatrics, 117(1), pp. 15-21.

[6] Garde, S., Knaup, P., Hovenga, E. J., and Heard, S., 2007. "Towards semantic interoperability for electronic health records". Methods of Information in Medicine, 46(03), pp. 332-343.

[7] Rossi, L., Belli, A., De Santis, A., Diamantini, C., Frontoni, E., Gambi, E., Palma, L., Pernini, L., Pierleoni, P., Potena, D., Raffaeli, L., Spinsante, S., Zigaretti, P., et al., 
2014. "Interoperability issues among smart home technological frameworks". In 2014 IEEE/ASME 10th International Conference on Mechatronic and Embedded Systems and Applications (MESA), IEEE, pp. 1-7.

[8] Kierkegaard, P., 2011. "Electronic health record: Wiring Europes healthcare". Computer law \& security review, 27(5), pp. 503-515.

[9] FSE, L. G., 2015. Framework dei servizi di integrazione con l'infrastruttura fse di regione marche.

[10] Lehner, W., and Sattler, K.-U., 2010. "Database as a service (DBaaS)". In IEEE International Conference on Data Engineering, IEEE, pp. 1216-1217.

[11] Munir, K., 2015. "Security model for cloud database as a service (DBaaS)". In International Conference on Cloud Technologies and Applications, IEEE, pp. 1-5.

[12] Frontoni, E., Baldi, M., Zingaretti, P., Landro, V., and Misericordia, P., 2014. "Security issues for data sharing and service interoperability in ehealth systems: the nu.sa. test bed". In 2014 International Carnahan Conference on Security Technology (ICCST), no. 6986999, IEEE, pp. 1-6.

[13] Dai, J., Parry, D. M., and Krahn, J., 1997. "Transcutaneous bilirubinometry: its role in the assessment of neonatal jaundice". Clinical Biochemistry, 30(1), pp. 1-9.

[14] Lilien, L. D., Harris, V. J., Ramamurthy, R. S., and Pildes, R. S., 1976. "Neonatal osteomyelitis of the calcaneus: complication of heel puncture". The Journal of Pediatrics, 88(3), pp. 478-480.

[15] Triantafillou, P., 2017. "Making electronic health records support quality management: A narrative review". International Journal of Medical Informatics, 104, pp. 105-119.

[16] Cenci, A., Liciotti, D., Frontoni, E., Mancini, A., and Zingaretti, P., 2015. "Non-contact monitoring of preterm infants using rgb-d camera". In ASME 2015 International Design Engineering Technical Conferences and Computers and Information in Engineering Conference, American Society of Mechanical Engineers, pp. V009T07A003V009T07A003.

[17] Cenci, A., Liciotti, D., Frontoni, E., Zingaretti, P., and Carnielli, V. P., 2017. "Movements analysis of preterm infants by using depth sensor". In Proceedings of the 1st International Conference on Internet of Things and Machine Learning, ACM, p. 12. 